\title{
Desigualdad laboral y educativa de la población del Estado de México: un análisis de condición migratoria por sexo
}

\author{
José Antonio Soberón Mora, Alfonso Mejía Modesto \\ y Hugo Montes de Oca Vargas
}

Universidad Autónoma del Estado de México

\section{Resumen}

El documento aborda la migración interna hacia el Estado de México en los últimos veinticinco años, a partir de cohortes quinquenales que inician en los quince y finalizan en los veinticinco años de edad. El objetivo de este trabajo se enfoca en responder las siguientes incógnitas 1. ¿Existe desigualdad entre los inmigrantes y los residentes del Estado de México al momento de insertarse en el mercado laboral y de acceder a la educación formal considerando los grupos de edad definidos y el sexo? 2. De existir desigualdad ¿cómo incide esta entre inmigrantes y residentes en la búsqueda de empleo como en la permanencia escolar?

Palabras clave: migración interna, educación, trabajo, desigualdad.

Labor and educational inequality of the population in the State of Mexico: an analysis of immigration status and gender

\begin{abstract}
The document addresses internal migration to the State of Mexico in the last twenty-five years, based on five-year cohorts that begin at fifteen and end at twenty-five years of age. The objective of this work is to answer the following questions: 1 . Is there inequality between immigrants and residents of the State of Mexico at the time of entering the labor market and accessing formal education considering the defined age groups and sex? 2. If there is inequality, how does this affect immigrants and residents in the search for employment as well as in school permanence.
\end{abstract}

Keywords: Internal migration, education, work, inequality.

Artículo recibido el 14 de abril de 2019 y aprobado el 10 de diciembre de 2019. 


\section{INTRODUCCIÓN}

$\mathrm{L}$

a migración en el Estado de México es un fenómeno sumamente importante desde la perspectiva demográfica, en la mayoría de los casos los inmigrantes que llegan a la entidad tienen como motivo migratorio la búsqueda de mejores condiciones de vida, es decir, un mejor empleo, así como para alcanzar mayores posibilidades para acceder a la educación superior para ellos y sus hijos. Es por ello que el objetivo de este trabajo se enfoca en responder las siguientes incógnitas 1. ¿Existe desigualdad entre los inmigrantes y los residentes del Estado de México al momento de insertarse en el mercado laboral y de acceder a la educación formal considerando distintos grupos de edad y sexo? 2. De existir desigualdad ¿cómo incide esta entre inmigrantes y residentes en la búsqueda de empleo como en la permanencia escolar?

Para responder estas preguntas de investigación, se desarrolla un marco conceptual sobre la migración y la desigualdad y se procesa la información de los microdatos de los censos más recientes 1990, 2000, 2010 y la Encuesta Intercensal de 2015 para concluir con los hallazgos y su discusión.

La migración ha sido estudiada desde varias perspectivas. Por ejemplo, Partida y Martínez (2006) estudian los flujos migratorios durante el periodo 1955 a 2005. En su trabajo elaboran tasas de migración interestatal por entidad, también origen y destino de los inmigrantes a nivel estatal. En el análisis de los datos que presentan, llama la atención la marcada disminución de inmigrantes interestatales entre el último quinquenio del siglo XX y el primero del siglo XXI. Este tipo de trabajos se enfocan principalmente a la descripción del volumen y dirección de los flujos migratorios. ${ }^{1}$

Otros proyectos han revisado el papel que ha jugado la migración interna y destacan la participación que ha tenido el crecimiento económico como la relación que prevalece entre este y la movilidad de

1 Se da el nombre de migración o movimiento migratorio al desplazamiento, con traslado de residencia de los individuos desde un lugar de origen o lugar de partida, a un lugar de destino o lugar de llegada y que implica atravesar los límites de una división geográfica. Diccionario Demográfico Multilingüe, Español segunda edición 1982 http://www. demopaedia.org/tools/spip.php?page=generate_dictionary\&edition=es-ii\&format=ht$\mathrm{ml}$, recuperado el 18 de diciembre de 2018. 
las personas en busca de fortalecer sus condiciones económicas como laborales (Arroyo, 1989; Arroyo y Olmos, 1998).

Por otro lado, existen investigaciones dirigidas a recuperar el perfil de los inmigrantes y sus cambios en distintos momentos de la migración en México. El trabajo de Ana María Chávez (1999) describe elementos que perfilan las características de los inmigrantes respecto a los no inmigrantes, a partir de variables como el nivel de escolaridad, sexo, grupos quinquenales o la relación de parentesco, en tres regiones de la República Mexicana: la Frontera Norte, Centro (excluyendo Distrito Federal) y el Distrito Federal (ahora Ciudad de México).

Adicionalmente, con información del censo del año 1990, la misma autora reporta información para el centro de la República Mexicana sin incluir el Distrito Federal, pero sí el Estado de México. En este caso, la comparación proporcional permite visualizar que hasta la secundaria completa se distinguen valores más elevados de quienes son inmigrantes. Al llegar a los sujetos con preparatoria completa la diferencia supera el 50 por ciento (el comportamiento de estos porcentajes permitiría imaginar un par de rectas con pendientes que tienden a la intersección en las personas con primaria completa). No sucede lo mismo con el Distrito Federal donde la información es menos estable e impide identificar una tendencia clara (Tabla 1).

Los datos presentados para el centro de México retoman el nivel de escolaridad de los sujetos a nivel general y no exploran, como en nuestro caso, la comparación entre inmigrantes y residentes (también llamados no migrantes) de las cohortes específicas. Como resultado se detecta que la población inmigrante tiene más elevados niveles de escolaridad lo que puede ser reflejo de: a) Haber tomado todos los rangos de edad, b) Encontrar una mayor influencia de los jefes de hogar o c) Notar la influencia de la población económicamente activa, elementos que son retomados en las aproximaciones al Capital Humano (Pott et al., 2008) (ver Tabla 1).

En fecha más reciente, se han realizado trabajos sobre la migración interna que ha buscado establecer si el crecimiento económico va ligado a los movimientos poblacionales o si estos determinan al primero, ya que buena parte de ellos se han enfocado a describir esta liga o relación a partir de la observación de los eventos que ya han sucedido (Delgado y Márquez, 2007). De esta forma, se han construido explicaciones sobre la operación de ambas variables y de la participación de otras que tuviesen que ver con el esclarecimiento de los factores que definen 
la migración. La mayor parte de estos estudios han centrado su análisis en fuentes de información de tipo transversal, en donde se observa el comportamiento de algunas variables seleccionadas en un momento específico de tiempo.

Tabla 1. Nivel de escolaridad de la población no migrante e inmigrante, 1990 (migración reciente, porcentajes), México

\begin{tabular}{lccccr}
\hline & \multicolumn{2}{c}{$\begin{array}{c}\text { Centro sin el DF } \\
\text { o Ciudad de México }\end{array}$} & \multicolumn{2}{c}{$\begin{array}{c}\text { Distrito Federal } \\
\text { (Ciudad de México) }\end{array}$} \\
\cline { 2 - 6 } & No migrantes & Inmigrantes & No migrantes & Inmigrantes \\
\hline Total & 100 & 100 & 100 & 100 \\
\hline Sin instrucción & 15.3 & 7.0 & 5.2 & 6.6 \\
Primaria incompleta & 22.8 & 14.1 & 11.4 & 14.4 \\
Primaria completa & 20.9 & 20.1 & 18.0 & 21.8 \\
Secundaria incompleta & 7.0 & 8.7 & 9.3 & 7.4 \\
Secundaria completa & 13.8 & 16.3 & 15.9 & 16.3 \\
Preparatoria o más & & & & 40.2 & 33.4 \\
estudios & 20.2 & 33.7 & & & \\
\hline
\end{tabular}

Fuente: Chávez (1999, Cuadro 4.1).

Sobrino (2010) presentó un análisis de la migración reciente en el periodo que abarca de 1930 hasta 2005. Para determinar el volumen de la migración absoluta, para esto se auxilia de la información existente de los censos de población a partir del año 1900. En su trabajo revisa algunos conceptos como el de migración interurbana y los factores que la explican. Para este autor, los cuatro factores esenciales que explican la migración son: I) Mercado de trabajo, II) Calidad de vida, III) Mercado de vivienda, y IV) Ciclo de vida familiar.

Otros estudios sobre migración recuperan variables que se han evaluado con menos frecuencia, como los niveles de violencia, las condiciones urbanas o la calidad de vida percibida (Rodríguez y Busso, 2009). Los abordajes temáticos también examinan aspectos que tocan la migración interna de retorno por motivos de salud (Lu y Qin, 2014).

\section{Desigualdad}

Un componente esencial de los estudios de migración interna que menos se ha examinado directamente es la desigualdad, no obstante, frecuentemente se abordan a partir de las diferencias en donde se 
persigue evaluar la intervención de variables específicas y cuyo objetivo final sea detectar estas diferencias ya sea en distintos momentos en el tiempo o entre componentes. La desigualdad o diferencia se traduce en las posibilidades de analizar las dos categorías de actividad que se examinan en este documento, la educación y el trabajo. Ambas vistas desde otras aproximaciones teóricas en los trabajos mencionados con anterioridad.

Para aclarar este postulado partiremos que en la actualidad tenemos una sociedad cada vez más desigual. De acuerdo con Atkinson (2016), la desigualdad puede ser de dos tipos:

a) La desigualdad de oportunidades. Se refiere a que todas las personas deberían tener el mismo punto de arranque.

b) La desigualdad de resultados. Esta se relaciona mucho con las actividades redistributivas. Para esto es fundamental la igualdad competitiva y la no competitiva de oportunidades. La desigualdad de oportunidades del futuro se asocia a la desigualdad de hoy. Entonces los beneficiados de la desigualdad de hoy pueden transmitir una ventaja injusta a sus hijos en el futuro (como es el caso del análisis por cohorte). Por lo que si nos preocupa la desigualdad de resultados del futuro nos debe preocupar la desigualdad de resultado del presente.

A continuación, se ejemplifica la desigualdad de oportunidades y la desigualdad de resultados en el acceso a la educación y el empleo. En el contexto liberal la educación es vista como la clave para mantenerse al día en la innovación y evitar la exclusión de los jóvenes al nuevo mundo que demandaba nuevos conocimientos para el empleo.

En la actualidad, entre las generaciones más jóvenes, cada vez son más personas que alcanzan un nivel educativo mayor que la media, no obstante, esto ha estado acompañado de una disminución del poder para brindar la llamada movilidad ascendente a las personas. Esto es mover sus condiciones de vida hacia mejores y mayores ingresos, así como seguridad económica en un sentido amplio.

En un ámbito más próximo y desde la perspectiva liberal, la enseñanza que se imparte en las universidades requiere de tantas innovaciones tecnológicas y científicas que resulta cada vez más costosa. Ante esta situación se argumenta que no es justo que los hijos de familias pudientes no paguen por la educación de sus hijos. Es decir, cuando un joven accede a la educación gratuita y cuenta con recursos 
económicos suficientes, ocupa recursos públicos, frecuentemente escasos que bien podrían favorecer a población con mucho menos acceso a ellos. Por otro lado, también desde la perspectiva liberal, sería injusto que un joven se viera excluido por razones económicas a acceder a mejores instituciones, por lo que es necesario establecer el sistema de apoyo del Estado al sistema educativo con mayor demanda y un sistema de becas que apoye la igualdad de oportunidades (Vargas, 2018).

En un estudio reciente Huerta (2012) comparó los casos mexicano y chileno para observar el peso de la riqueza de origen y la escolaridad sobre el bienestar socioeconómico del niño. Se trata de dos dimensiones que contribuyen a perpetuar el ciclo de reproducción de la desigualdad, los padres con más recursos obtienen más credenciales educativas con lo cual generan ambientes más propicios para un mayor desarrollo educativo y económico de la progenie, una brecha que se origina desde edades tempranas. El sistema de educación pública tiene como fin romper las inercias entre orígenes y destinos y permitir que cualquier persona, sin importar su condición social de partida, pueda acceder a la educación solamente con base en su propio esfuerzo y talento (Huerta, 2012).

Frente al argumento liberal, se ha realizado investigación sobre la relación educación y pobreza. De acuerdo con Iliana Yaschine (2015) a finales de la década de 1960 a partir del trabajo de Blau y Duncan (en Yaschine, 2015) se planteó un modelo explicativo del proceso de estratificación, sobre la hipótesis liberal, que postulaba que el mérito sustituiría progresivamente al origen socioeconómico como determinante de la estratificación social. Analizando el peso relativo de los factores adscritos (definidos por nacimiento) y los no adscritos (o meritocráticos), algunos resultados de estas investigaciones señalan que la escolaridad es la más importante de las variables estudiadas, no obstante, el conjunto de factores adscritos ejerce también una influencia de magnitud notable en la estratificación ocupacional de estos individuos. Adicionalmente se ha encontrado que los factores relacionados con el origen socioeconómico ejercen mayor efecto entre los hombres en comparación con las mujeres.

Por su parte, Atkinson (2016) señala que la importancia de la igualdad de oportunidades radica en los resultados obtenidos. Es decir, cuando estos se convierten en la privación de los medios necesarios para alcanzar las metas propuestas. Otra razón radica en distinguir entre 
igualdad competitiva y no competitiva de oportunidad, la oportunidad no competitiva de satisfacer proyectos de vida independientes. La tercera razón es porque la desigualdad afecta directamente a la igualdad de oportunidad. En la temporalidad, este autor se refiere a que la desigualdad de resultados en la generación actual es la fuente de la ventaja injusta recibida por la próxima generación (Atkinson, 2016).

\section{El empleo del liberalismo al neoliberalismo}

Desde un acercamiento más económico, el liberalismo previo a la Segunda Guerra Mundial rechazaba a toda costa el intervencionismo del Estado. Pero en décadas más recientes, el neoliberalismo reclama la participación del Estado más allá de los mecanismos autoreguladores del mercado y la fe en la justicia de los contratos entre particulares que supuestamente son iguales, lo cual supone un marco jurídico que permita las condiciones óptimas para que la competencia o rivalidad satisfagan el interés colectivo (Laval y Dardot, 2013).

Como menciona Marina (2010: 123):

la economía neoliberal descansa en la imagen de un yo humano autárquico. Presupone que los individuos pueden dominar, ellos solos, la totalidad de sus vidas, y que obtienen y renuevan su capacidad de acción de su propio interior.

De esta manera, frente a las transformaciones económicas han ocurrido transformaciones en el mundo laboral, y por supuesto en el educativo, reflejándose directamente las oportunidades de ingreso a los respectivos sistemas.

\section{Del Fordismo al Toyotismo}

Uno de los planteamientos que se han formulado para explicar la transformación del mundo del trabajo durante las últimas décadas, es la transición del Fordismo al Posfordismo, también llamado Toyotismo mismas que se caracterizan (Aguilar y Zepeda, 2015):

\section{A. Fordismo}

-Producción masiva y altamente estandarizada.

- Fuerte división del trabajo y clara separación tanto entre las labores de ejecución como de concepción dentro de la empresa. 
- Papel dominante de la gran empresa tanto en la producción como en la innovación.

- Uso de maquinaria especializada y predominio de fuerza de trabajo no calificada.

-Economías de escala y cadena de montaje.

-Petróleo barato como factor energético clave.

B. Toyotismo

-Diversificación de la demanda por consumo y, por lo tanto, menor estandarización de los productos.

- Flexibilidad en las tecnologías y en la organización industrial, debido al uso de la electrónica como factor clave.

- Fuerza de trabajo de mayor calificación y polivalente.

-Descentralización de funciones dentro de la gran empresa, lo que lleva a una desintegración vertical.

-Papel más importante de las Pequeñas y Medianas Empresas (Pymes) debido a la subcontratación y los desprendimientos empresariales (spin-offs).

- Predominio de las economías de alcance sobre las de escala.

- Mayor importancia de las instituciones como actores del desarrollo económico tanto en el nivel mundial como en el regional y local.

Previamente se ha revisado las aportaciones teóricas desde el punto de vista de la economía. A continuación nos enfocamos a recuperar desde la perspectiva sociológica y como opción al neoliberalismo, la perspectiva teórica de la sociedad de riesgo. La cual es sumamente amplia y rica de matices que han sido poco explorados en Latinoamérica.

Hoy en día no vivimos en el mundo liberal o en el mundo socialista, vivimos en la sociedad del riesgo. Desde hace unas décadas el mundo occidental ha transitado por un proceso que puede denominarse como de la sociedad laboral a la sociedad de riesgo, en categorías propuestas por Ulrich Beck (1997). Este proceso ha dado lugar a una creciente pérdida de la certidumbre y la exclusión para gran parte de la población, esto no obstante los niveles de estudio que se tengan.

En la sociedad de la segunda modernidad en el mundo desbocado como lo llamaría Giddens (2000). El tener una baja escolaridad te 
condena en la mayoría de los casos a un empleo mal remunerado y poco satisfactorio en todos sentidos.

\section{La sociedad del riesgo}

Así se pueden distinguir entre dos riesgos: el riesgo externo, lo que afecta al individuo constantemente; y el riesgo manufacturado, donde la ciencia y la tecnología están involucradas. El riesgo externo está comprendido en términos concretos por todos aquellos eventos que pueden alcanzar a las personas inesperadamente, aunque ocurren con regularidad y frecuencia en una población completa y, por tanto, son predecibles y susceptibles de asegurarse. Es aquí donde se ubica el surgimiento del Estado Benefactor, donde protegerse del riesgo es una necesidad de seguridad colectiva y no privada. En una sociedad que vive después de la naturaleza y la tradición, es que surge el riesgo manufacturado, es decir, el riesgo creado por la progresión del desarrollo humano, en especial por el crecimiento de la ciencia y la tecnología. Este riesgo se extiende a casi todas las dimensiones de la vida humana (Giddens, 1998).

Para casi todos, ya no se sostiene más la idea de que el hijo del zapatero sería zapatero. Los jóvenes han sido lanzados a la libertad de ser lo que quieran, pero también a experimentar los riesgos como algo individual. Si antes existían procesos de formación para el trabajo bien definidos, hoy en día estos están casi perdidos, no hay gremios o familias que sostengan los procesos de formación para el trabajo. Incluso las mujeres se han incorporado al mercado laboral participando también de todos los riesgos. El nicho de solo de amas de casa y madre se ha ido desdibujando para la mayoría.

Las oportunidades, los peligros y las incertidumbres biográficas, que antes estaban predefinidas dentro de la asociación familiar o de la comunidad rural, o a tenor de las normativas de los estados o clases asistenciales, deben ahora percibirse, interpretarse, decidirse y procesarse por los propios individuos. Las consecuencias - tanto las oportunidades como las cargas - pasan ahora a las personas que, naturalmente, frente a la complejidad de las interrelaciones sociales, se ven a menudo incapaces de tomar las decisiones necesarias con el debido fundamento, ponderando los intereses, la moral y las posibles derivaciones (Beck y Beck-Gernsheim, 2003: 42).

Según el enfoque teórico de la sociedad del riesgo, el mundo es más abierto y contingente de lo que podría sugerir cualquier concepción 
clásica de la sociedad moderna. Los eventos que no existen (todavía) tienen una gran influencia en nuestros asuntos y acciones presentes. Por tanto, los elementos revisados previamente y que abordan la transformación del Fordismo al Toyotismo se pueden vincular claramente a la sociedad de riesgo. Cada vez las personas se encuentran más amenazadas por la precarización del empleo que deviene de la subcontratación y de la incertidumbre laboral.

En México, alcanzar la mayoría de edad legal (18 años) también significa para muchas personas abandonar la escuela. Solo la mitad, o menos, continúan asistiendo a ésta, por lo que establece un límite importante para en el mejor de los casos alcanzar a concluir la educación media superior. Vemos dos tendencias en apariencia contradictorias. Por un lado, el porcentaje de hombres que abandonan la escuela es mayor en comparación con las mujeres. Pero después de los 20 años el porcentaje de hombres que continúa con los estudios comienza a alinearse con el de las mujeres, no obstante, la participación de ambos desciende aceleradamente.

Resalta de manera muy notable el contraste entre las tasas de matriculación y la eficiencia terminal en algunas entidades. Destaca que la eficiencia terminal más baja en nivel medio superior corresponde a la Ciudad de México. Con un valor cercana al 50 por ciento. Es decir sólo la mitad de los estudiantes que entran a preparatoria terminan. Pero esta condición no es única, como se verá más adelante, con los datos utilizados, además de la deserción, el ingreso a los distintos niveles educativos es mermado en edades propias para la continuidad de la formación académica (INEGI, 2019).

\section{MATERIALES Y MÉTODOS}

Como fuente de información se usaron los microdatos de los Censos de Población y Vivienda 1990, 20002010 y la Encuesta Intercensal 2015. La inclusión de variables migratorias en la Encuesta Intercensal 2015 no es exclusivo de este evento y para el proyecto que se presenta, se ha retomado la variable que capta la migración reciente desde el censo del año 1990. De esta manera los censos de población utilizados captan la migración en dos momentos, lugar de nacimiento y lugar de residencia cinco años antes. Se ha elegido la variable migración por residencia cinco años atrás, porque capta la migración "reciente" y permite 
tener un acercamiento al comportamiento migratorio comparado en quinquenios. $^{2}$

Se realizó el procesamiento estadístico correspondiente a la información de los cuestionarios ampliados de los censos poblacionales de los años 1990, 2000, 2010 y la Encuesta Intercensal del año 2015. Los tamaños de las muestras fueron definidos de manera probabilística y con un diseño muestral probabilístico que permite obtener resultados estadísticamente robustos a nivel estatal y municipal. En particular el tamaño de la muestra de la Encuesta Intercensal 2015 supera casi en el doble a la muestra del censo 2010.

A continuación, se muestran las respectivas proporciones de inmigrantes recientes y de toda la vida (que nacieron en la entidad no obstante esta variable no se utiliza) como de las poblaciones totales en cada uno de los respectivos periodos. Al examinar la información que se presenta, se hace evidente la participación de la entidad como una de las principales receptoras de migrantes nacidos en otros estados (Tabla 2).

Tabla 2. Inmigrantes internos del Estado de México según fuente (Cifras en porcentaje), 1990-2015

\begin{tabular}{lrrrr}
\hline & Censo 1990 & Censo 2000 & Censo 2010 & Encuesta 2015 \\
\hline Interno reciente* & 21.1 & 17.2 & 14.3 & 12.5 \\
Toda la vida & 40.2 & 42.1 & 38.4 & 34.3 \\
Población total & $9,793,720$ & $13,058,570$ & $15,123,304$ & $16,187,608$ \\
\hline
\end{tabular}

* Población de 5 o más años. Proporciones a partir del total.

Fuente: INEGI: Microdatos de los censos poblacionales de los años 1990, 2000, 2010 y Encuesta Intercensal 2015.

Por ende, los sujetos a que nos referimos fueron seleccionados una vez que: a) Dijeron haber radicado en una entidad diferente a la de la entrevista cinco años antes (condición inmigrante) o ser residente si se encontraba residiendo en la misma entidad, b) Su edad se encontraba dentro de los quinquenios (también denominados rangos de edad o cohortes) 15-19 $\mathrm{o}_{5} \mathrm{~N}_{15}, 20-24 \mathrm{o}_{5} \mathrm{~N}_{20}$ años cumplidos.

2 La selección de los sujetos inmigrantes considera a aquellos que cinco años antes radicaban en un lugar distinto al de residencia. La unidad de medida quinquenio coincide, en este caso, con la agregación de las edades en quinquenios tal y como se presentan en los correspondientes cuadros. 
Para comparar la desigualdad laboral y educativa de la población en el Estado de México, se procedió a dividir en grupos como se muestra en la Tabla 3.

Tabla3. Comparación de cada una de las cohortes por la variable de comparación condición de residencia (residentes-inmigrantes) para cada una de las mediciones.

\begin{tabular}{cccccc}
\hline & Trabajaron & \multicolumn{3}{c}{ Estudiaron } \\
Residentes & & Inmigrantes & Residentes & & Inmigrantes \\
\hline${ }_{5} \mathrm{~N}_{15}$ & vs & ${ }_{5} \mathrm{~N}_{15}$ & ${ }_{5} \mathrm{~N}_{15}$ & vs & ${ }_{5} \mathrm{~N}_{15}$ \\
${ }_{5} \mathrm{~N}_{20}$ & vs & ${ }_{5} \mathrm{~N}_{20}$ & ${ }_{5} \mathrm{~N}_{20}$ & vs & ${ }_{5} \mathrm{~N}_{20}$ \\
\hline
\end{tabular}

Fuente: Elaboración propia.

\section{RESULTADOS}

Al examinar los resultados obtenidos se confirma la hipótesis planteada en el enfoque conceptual sobre la exclusión de grupos sociales. Se observa entonces que los estudiantes de las edades quinquenales seleccionadas reflejan un comportamiento desigual a partir de la observación de la variable de comparación, condición de residencia y sexo.

En un primer momento se examinan y procesan los resultados obtenidos a partir del censo del año 1990. El primero de los quinquenios mostrados, incluye las edades de $15,16,17,18$ y 19 años $\mathrm{o}_{5} \mathrm{~N}_{15}{ }^{(1990)}$, se destaca ya la presencia de una diferencia entre los sujetos que trabajan y que estudian que además son examinados por medio de la variable condición de residencia. ${ }^{3}$

$\mathrm{Al}$ examinar el quinquenio de la cohorte ${ }_{5} \mathrm{~N}_{15}$, esta es la que reporta los valores que discriminan con mayor intensidad ya sea que estudien o trabajen. Los sujetos que dijeron estudiar reflejan 8.8 puntos porcentuales de diferencia de acuerdo con la información del censo del año 1990, resultado que reporta una $p<.01$ al aplicar la respectiva prueba de contraste (46.7 - 37.9) (Tabla 3). ${ }^{4}$

Al continuar con la cohorte ${ }_{5} \mathrm{~N}_{15}$ hay otros valores igualmente elevados en los tres periodos restantes. La información de años 2000, 2010 y 2015 proporciona las respectivas diferencias, entre inmigrantes

3 La notación se utiliza para precisar edades exactas. De este modo ${ }_{5} \mathrm{NF}_{15}$ corresponde a la cohorte femenina que inicia en 15 años, pero todavía no cumple 20 años.

4 Al aplicar la prueba Chi cuadrada se tomaron las variables por pares para su análisis. En el primer caso se incluyeron en el análisis sexo y condición migratoria del quinquenio ${ }_{5} \mathrm{~N}_{15}$, en el segundo análisis estudia y condición migratoria del mismo quinquenio. Lo mismo para el quinquenio ${ }_{5} \mathrm{~N}_{20}$. 
y residentes, de quienes estudian 12.2, 12.9 y 13.3. Esta información pone en evidencia que, no obstante, se percibe un claro incremento en las respectivas diferencias, de los sujetos que dijeron estudiar durante los cuatro periodos. Por otro lado, y es menos evidente, se incrementa también la participación de quienes dijeron estudiar durante las cuatro mediciones. El respectivo porcentaje de los residentes pasó de 46.7 en 1990 a 62.0 en el año 2015, el de los inmigrantes paso de 37.9 a 48.7 (Tabla 4).

La observación anterior se invierte relativamente porque los valores de quienes trabajan disminuyen sí, pero con menor velocidad. Los residentes pasaron de 25.1 a 17.9 y los inmigrantes de 32.8 a 24.7. Esta categoría también muestra diferencias entre residentes y migrantes como en el caso del año 2000 en donde se obtiene $11.4 \mathrm{si}$ restamos 42.4 menos 31.0 (Tabla 4).

La variable "trabajó" de la cohorte ${ }_{5} \mathrm{~N}_{15}$ ha ido perdiendo espacio en el transcurso de las mediciones, mismo que en parte es el espacio que ha ganado la categoría "estudió". Esta ganancia es más notoria en la primera de las cohortes y eso es más visible a diferencia de lo que sucede en la segunda cohorte, no obstante, ambas muestran una tendencia a mantener las distancias entre ambas categorías y por tipo de residencia.

Tabla 4. Personas de la cohorte ${ }_{5} \mathrm{~N}_{15} \mathrm{y}_{5} \mathrm{~N}_{20}$ dijeron trabajar o estudiar en el Estado de México, 1990-2015 (Porcentajes)

\begin{tabular}{rrrrrrrrr}
\hline & \multicolumn{3}{c}{${ }_{5} \mathrm{~N}_{15}$} & & \multicolumn{3}{c}{${ }_{5} \mathrm{~N}_{20}$} \\
\\
\hline
\end{tabular}

Fuente: Elaboración propia a partir de los censos de 1990, 2000, 2010 y Encuesta Intercensal 2015.

* Resultados con $p<.01$ al aplicar Chi2 con 3 y 8 grados de libertad con ponderación relativizada, para obtenerla se dividió la base total ponderada sobre la base no ponderada, el cociente obtenido se multiplica por el factor original.

Las diferencias que se han descrito a partir de la Tabla 4 se muestran de forma gráfica, y enfatizan las respectivas proporciones entre los 
sujetos que trabajan y los que residen. Si tomamos en cuenta que la cohorte ${ }_{5} \mathrm{~N}_{15}$ podría estar estudiando en su totalidad, únicamente el 46.7 por ciento de los sujetos entrevistados en el año 1990 lo hacían. Esta condición se encuentra asociada a la necesidad de trabajar de las familias del Estado de México en donde, de acuerdo con el Consejo Nacional de Evaluación de la Política de Desarrollo Social (CONEVAL, 2018), para el año 1990 el 48.3 por ciento de la población de la entidad se encontraba en condiciones de pobreza patrimonial. Sobre la misma cohorte 1, es visible que para el año 2015 el 38 por ciento de la población residente ya no cumplía con actividades educativas, y con ello es notorio lo que ya se ha venido destacando, la proporción de los sujetos que estudian y son migrantes es menor. Esta desigualdad es notoria en la primera de las cohortes, pero disminuye una vez que exploramos a los sujetos de la cohorte ${ }_{5} \mathrm{~N}_{20}$ o que tienen entre $20 \mathrm{y}$ 24 años cumplidos, esta condición es independiente de la fecha que se examine y el valor de diferencia más elevado entre los estudiantes sean residentes o migrantes es 8.3 (Figura 1).

Figura 1. Personas de la cohorte ${ }_{5} \mathrm{~N}_{15} \mathrm{y}_{5} \mathrm{~N}_{20}$ dijeron trabajar o estudiar en el Estado de México, 1990-2015 (Porcentajes)

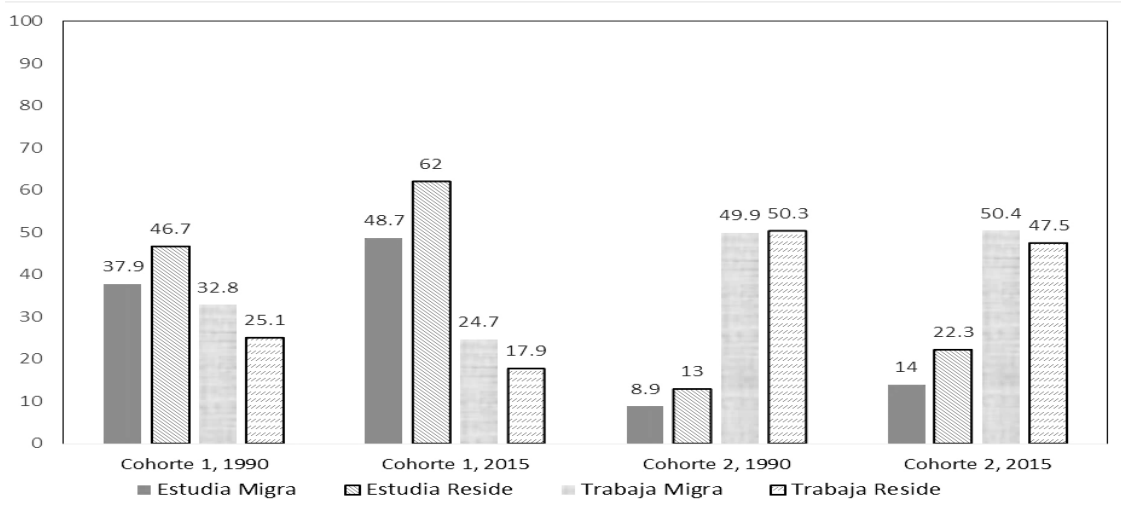

Fuente: Elaboración propia con base en la Tabla 4.

\section{Diferencia por sexo, cohorte ${ }_{5} \mathbf{N}_{15}$.}

De manera general, podemos afirmar que el comportamiento de ambos sexos respecto de las variables que se vienen examinando, se ha mantenido. Al realizar el análisis por sexo la proporción de hombres inmigrantes que mencionaron trabajar en la medición de 1990 fue 
de 44.3 por ciento, misma que ha sido mayor que la de residentes la cual llega tan solo a 34.7. A pesar de ello, para la medición de 2015 ambas proporciones se reducen y los migrantes hombres que trabajan representan el 33.9 por ciento y los residentes 25.0 por ciento. Es notorio que la variable más discriminadora se ubica en quienes dijeron estudiar. Pero este efecto no es exclusivo de los hombres, el segmento femenino ${ }_{5} \mathrm{NF}_{15}$, al igual que el masculino ${ }_{5} \mathrm{NM}_{15}$, incrementó su porcentaje con respecto a las personas que estudian y son migrantes de 35.2 a 46.1 por ciento, el caso de las residentes es el más radical porque no obstante se incrementó de 45.4 a 63.2 por ciento, también se incrementó casi al doble (46.1 y 63.2) la diferencia entre ambas categorías (Tabla 5 y Figura 2).

Tabla 5. Personas de la cohorte ${ }_{5} \mathrm{~N}_{15}$ dijeron trabajar o estudiar en el Estado de México por sexo, 1990-2015 (Porcentajes)

\begin{tabular}{rrrrrrrrrr}
\hline & \multicolumn{3}{c}{ Hombres } & \multicolumn{4}{c}{ Mujeres } \\
& \multicolumn{2}{c}{ Trabajó } & \multicolumn{2}{c}{ Estudió } & \multicolumn{2}{c}{ Trabajó } & \multicolumn{2}{c}{ Estudió } \\
& Reside & Inmigra & Reside & Inmigra & Reside & Inmigra & Reside & Inmigra \\
\hline $1990^{*}$ & 34.7 & 44.3 & 47.9 & 41.2 & 15.7 & 23.4 & 45.4 & 35.2 \\
$2000^{*}$ & 40.0 & 53.8 & 39.3 & 28.9 & 22.0 & 33.0 & 40.8 & 26.9 \\
$2010^{*}$ & 30.8 & 42.4 & 54.4 & 45.2 & 14.6 & 18.0 & 57.4 & 41.2 \\
$2015^{*}$ & 25.0 & 33.9 & 60.9 & 51.4 & 10.7 & 15.8 & 63.2 & 46.1 \\
\hline
\end{tabular}

Fuente: Elaboración propia a partir de los censos de 1990, 2000, 2010 y Encuesta Intercensal 2015.

* Resultados con $\mathrm{p}<.01$ al aplicar Chi 2 con 3 y 8 grados de libertad, ponderación relativizada.

Figura 2. Personas de la cohorte ${ }_{5} \mathrm{~N}_{15}$ dijeron trabajar o estudiar en el Estado de México por sexo, 1990-2015 (Porcentajes)

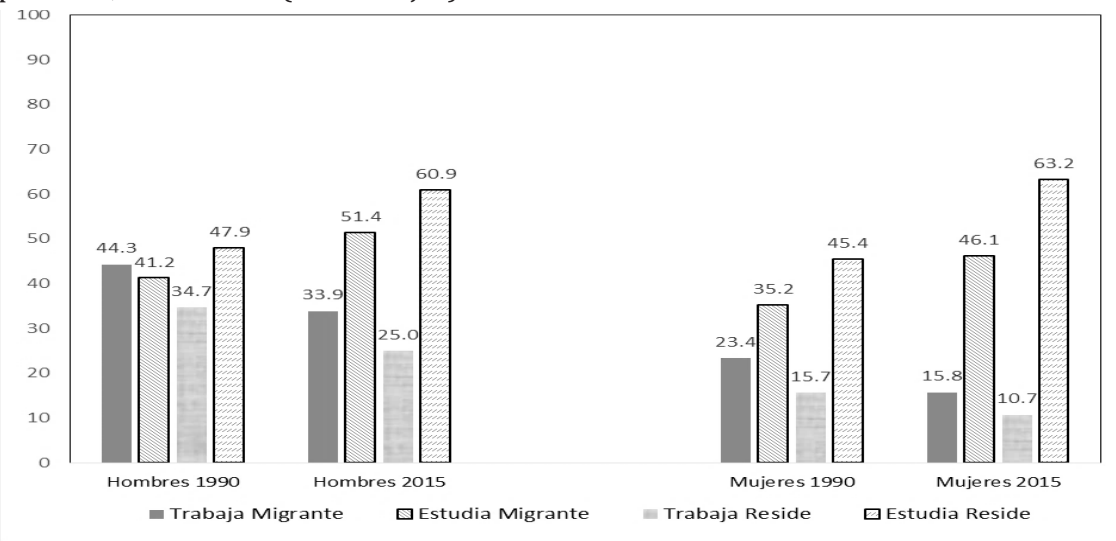

Fuente: Elaboración propia con base en la Tabla 5. 


\section{Diferencia por sexo, cohorte ${ }_{5} \mathbf{N}_{20}$.}

La información que se obtiene con respecto a la segunda cohorte ${ }_{5} \mathrm{~N}_{20}$, como en el caso de la primera, refleja condiciones de desigualdad. Para el año 2015 todavía eran más los hombres que estudiaban y eran residentes 22.7 por ciento, que los migrantes participaban con solo 16.0 por ciento. Esta condición también se ve reflejada entre las mujeres en que son residentes las cuales representan el 21.8 por ciento contra quienes son migrantes 12.4. Pero esa no es la diferencia más notoria, la participación de las mujeres en actividades asociadas a la atención del hogar es más de la mitad, en ello se ubican actividades como la atención de los hijos, atención de los padres, cuidados y atención del hogar, etcétera (Tabla 5 y Figura 3).

A pesar de encontrarnos con valores elevados de sujetos que trabajan o estudian, un porcentaje significativo de los hombres reporta no trabajar, de acuerdo con esta condición, las cifras que reporta la Universidad Nacional Autónoma de México (UNAM) muestran una tasa de desocupación para el primer trimestre de 2015 mayor a cuatro por ciento, cifra que en los últimos siete años no ha descendido del mismo cuatro por ciento (UNAM, s/f).

Tabla 6. Personas de la cohorte ${ }_{5} \mathrm{~N}_{20}$ dijeron trabajar o estudiar por sexo, Estado de México, 1990-2015 (Porcentajes)

\begin{tabular}{rrrrrrrrr}
\hline & \multicolumn{3}{c}{ Hombres } & \multicolumn{3}{c}{ Mujeres } \\
& \multicolumn{2}{c}{ Trabajó } & \multicolumn{2}{c}{ Estudió } & \multicolumn{2}{c}{ Trabajó } & \multicolumn{2}{c}{ Estudió } \\
\hline & Reside & Inmigra & Reside & Inmigra & Reside & Inmigra & Reside & Inmigra \\
$1990^{*}$ & 72.3 & 79.1 & 14.6 & 11.1 & 29.7 & 27.9 & 11.5 & 7.2 \\
$2000^{*}$ & 75.5 & 83.4 & 11.2 & 6.8 & 37.1 & 36.6 & 10.4 & 5.1 \\
$2010^{*}$ & 68.1 & 81.7 & 18.4 & 10.0 & 36.2 & 39.1 & 18.7 & 9.5 \\
$2015^{*}$ & 63.8 & 74.7 & 22.7 & 16.0 & 31.6 & 31.3 & 21.8 & 12.4 \\
\hline
\end{tabular}

Fuente: Elaboración propia a partir de los censos de 1990, 2000, 2010 y Encuesta Intercensal 2015.

* Resultados con $p<.01$ al aplicar Chi2 con 3 y 8 grados de libertad, ponderación relativizada.

Con la información analizada se encontró que el comportamiento de los porcentajes mantiene una tendencia, que procede generalmente hacia el alza en educación o hacia la baja en trabajo de acuerdo con las variables analizadas a través de las cuatro mediciones.

Este es el caso de los sujetos que trabajan, son inmigrantes y que pertenecen al quinquenio ${ }_{5} \mathrm{NF}_{15}$. La pendiente en bajada a lo largo de 
Figura 3. Personas de la cohorte ${ }_{5} \mathrm{~N}_{20}$ dijeron trabajar o estudiar por sexo, Estado de México, 1990-2015 (Porcentajes)

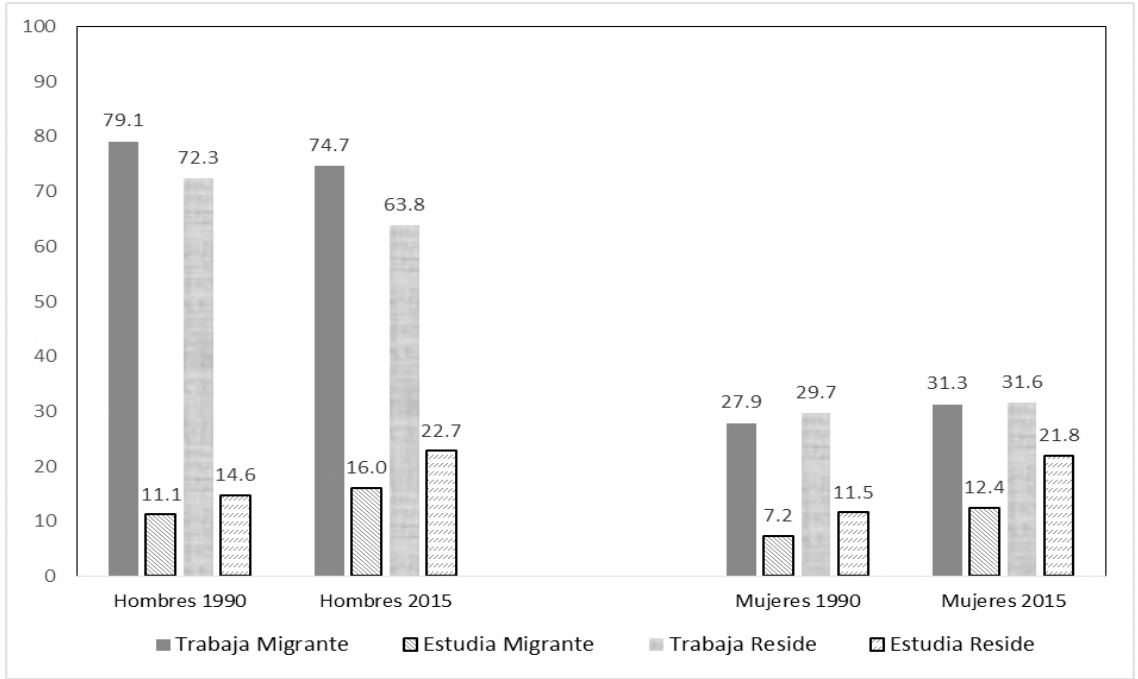

Fuente: Elaboración propia con base en la Tabla 6.

las mediciones es constante y tan sólo en la medición del año 2000 recupera un volumen considerable. Lo mismo se puede observar en los sujetos que reportaron ser residentes, el descenso es notorio con excepción de la medición del año 2000 (Tablas 4 a 6).

De forma inversa, los sujetos que estudian, tanto los inmigrantes como los residentes experimentan un incremento en su participación además de mostrar, al igual que en el caso de los que trabajan, un salto en la medición del año 2000 (Tablas 4 a 6). Además de esta estabilización, se observa un incremento, con un nivel bajo, de las personas que continúan sus estudios en la cohorte ${ }_{5} \mathrm{~N}_{20}$, tanto para hombres como para mujeres. Este incremento es más confuso en los hombres inmigrantes en donde no se puede marcar una tendencia. Es claro observar que, como se expuso en la parte teórica de este documento, el porcentaje de población que deja de estudiar se reduce drásticamente en proporciones que van de 45 por ciento a 15 por ciento al comparar los dos últimos quinquenios ${ }_{5} \mathrm{~N}_{25} \mathrm{y}_{5} \mathrm{~N}_{30}$ en las cuatro mediciones.

Al ver el comportamiento de las respectivas proporciones el lector se percata que los hombres que reportaron trabajar en este mismo quinquenio ${ }_{5} \mathrm{~N}_{15}$ mantienen un descenso en la participación 
de este factor a lo largo de las cuatro mediciones. Elemento cuyo comportamiento se refleja en otro inversamente relacionado que es la participación en los sujetos que reportaron estudiar. A pesar de esta condición, al examinar la siguiente cohorte, la presencia cada vez más numerosa de hombres que trabajan, comprueban una buena parte de los roles de género que ha reproducido la sociedad mexicana examinada por ahora a través de los datos del Estado de México. En otras palabras, el análisis por sexo de ambos factores de actividad muestra que para el quinquenio examinado ${ }_{5} \mathrm{~N}_{15}$ los hombres que trabajan siempre van a reportar niveles de participación más elevados que las mujeres en esta misma cohorte. Una vez que se tienen los resultados para estas dos actividades y categorías, y se suman, se comprueba la función del rol establecido al encontrar la mayor parte complementaria dentro de la categoría de actividades domésticas. Es fácil percatarse de que son las mujeres las que participan con mayor porcentaje.

\section{Discusión}

No todo el panorama es gris, las campañas tendientes a promover la igualdad de oportunidades tanto para hombres como para mujeres, ha provocado que para el año 2015 el porcentaje de la cohorte ${ }_{5} \mathrm{NF}_{15}$ residentes y con actividades educativas sea el más alto, por supuesto que incluso a la de los hombres. Cabe destacar que este incremento en actividades educativas ha disminuido otras actividades como las domésticas o incluso el grupo que trabaja. Esta condición, además de mostrar el incremento señalado, refleja una reducción en la desigualdad entre sexos extremadamente marcada en 1990.

No se puede negar el incremento en las respectivas proporciones de la gente que indicó estudiar cuando se comparan los años 1990 y 2015 principalmente de la cohorte ${ }_{5} \mathrm{NF}_{15}$, desafortunadamente este incremento no se distingue en el volumen de la siguiente cohorte lo que daría lugar a indagar la actividad que reportan las personas que no estudian. Las opciones posibles son principalmente que las personas dediquen su tiempo a actividades domésticas, al cuidado de personas o incluso a la ausencia de alguna otra actividad específica.

Respecto a la variable "actividad", si a la información censal nos remitimos, la información proporcionada por el Instituto Nacional de Estadística y Geografía (INEGI) de las personas entre 15 y 24 años evidencia un incremento entre las mediciones del año 2000, 2010 y 2015 , a nivel nacional tanto para hombres y mujeres, pero es notoria la 
condición de desigualdad que ha crecido cuando nos percatamos que el mayor incremento la tienen aquellas mujeres residentes y las más bajas corresponden a las que son migrantes (INEE, 2019).

Tampoco se puede negar que un grupo de población que ha contribuido, con su reducción, son las mujeres que trabajan en la primera cohorte, sin olvidar el volumen que reportó labores domésticas. En este caso el comportamiento de los hombres no distingue una tendencia.

Al encontrar independencia con respecto a las dos opciones (educación y empleo) de la variable examinada (actividad) nos percatamos además que pertenecen a un conjunto de posibilidades exhaustivas. Si el 40 por ciento trabaja y el 10 estudia, el restante 50 necesariamente debe estar clasificado bajo otro concepto.

La independencia se refiere a la diferencia matemática sencillamente, pero no a una independencia experimental en donde la manipulación de una variable independiente surte efecto en otra dependiente. El límite que establecemos en ambos procedimientos apenas y es perceptible y posiblemente la única diferencia en aplicar uno u otro (Chi cuadrada o regresión) obedezca a elementos conceptuales.

El análisis de las desigualdades a partir de variables sociodemográficas no debe dejar de lado la aproximación de las condiciones observadas, a partir de la figura "jefatura del hogar". Para este caso, el análisis de las condiciones de educación como de inserción en el trabajo se definiría dentro de los mismos grupos quinquenales, solo que en esta ocasión en lugar de comparar a los sujetos de manera directa y bajo la condición de residencia antes definida, se haría bajo la condición de residencia del jefe de familia, a partir de ello se realiza la cuantificación únicamente de los sujetos pertenecientes al hogar. Esta manera de analizar la información puede mostrar un nivel discriminativo distinto a lo previamente encontrado o profundizar en las diferencias.

Los contextos de desigualdad encontrados de la variable examinada se encuentran asociados a un conjunto de condiciones prevalecientes en un sistema socioeconómico configurado a partir de decisiones neoliberales. El aceptar la existencia de estas condiciones, limita las acciones que se pudiesen tomar con el objeto de alterar su representatividad. Si en determinado momento se tomara la iniciativa de incrementar el número de años que cursan los sujetos de las 
cohortes seleccionadas, se llegaría únicamente a eso, incrementar el número de años de escolaridad sin considerar el acceso a actividades laborales al término de aquella. Es decir, se requiere fomentar las oportunidades laborales de calidad para los egresados de educación media superior y superior. Sobre este particular, Atkinson (2016) retoma la importancia de observar los resultados que los sujetos tienen a partir de las oportunidades que se presentan y que en teoría serían iguales.

De acuerdo a los resultados presentados, es evidente la necesidad de distinguir las diferencias existentes entre las oportunidades educativas y laborales para la desigualdad presente y futura de las distintas generaciones que enfrentan la competencia global y un mercado laboral con condiciones cada vez más precarias.

\section{REFERENCIAS BIBLIOGRÁFICAS}

Aguilar, Javier y Roberto, Zepeda M. (2015). “Mundialización y tasa sindical en las sociedades contemporáneas". Análisis comparativo en países de América del Norte y Europa Occidental, Instituto de Investigaciones Sociales, Universidad Nacional Autónoma de México, México.

Arroyo Alejandre, Jesús. (1989). El abandono rural, Universidad de Guadalajara, México.

Arroyo Alejandre, Jesús y Edgar, Olmos Santamaría. (1998). "Políticas económicas y distribución territorial de la población en el occidente de México, 1960-1995", en: Arroyo Alejandre, Jesús (coord.), Economía regional y Migración. Cuatro estudios de caso en México, Universidad de Guadalajara, Asociación Mexicana de Población, A. C., y Juan Pablos (ed.), Guadalajara, México.

Atkinson, Antony. (2016). Desigualdad ¿qué podemos hacer? México, Fondo de cultura Económica.

Beck, U. (1997). "La reinvención de la política: Hacia una teoría de la modernización reflexiva", en Beck U. A. Giddens y S. Lash. Modernización reflexiva política, tradición y estética en el orden social moderno. Alianza Universidad, España.

Beck, Ulrich y Elisabeth, Beck-Gernsheim. (2003). La individualización. El individualismo institucionalizado y sus consecuencias sociales y políticas, España, Paidós.

Chávez Galindo, A. M. (1999). La nueva dinámica de la migración interna en México de 1970 a 1990. Cuernavaca: Centro Regional de Investigaciones Multidisciplinarias UNAM. 
CONEVAL. (2018). Estadísticas de pobreza en el Estado de México. Recuperado el 25 de enero de 2019 en https://www.coneval.org.mx/ coordinacion/entidades/EstadodeMexico/Paginas/pob_ingresos.aspx

Delgado, R. Márquez, H. (2007). "Teoría y práctica de la relación dialéctica entre desarrollo y migración". Migración y Desarrollo, 9, 5-25.

Diccionario Demográfico Multilingüe. (1982). Español segunda edición consultado el 18 de diciembre de 2018 en http://www.demopaedia.org/ tools/spip.php?page=generate_dictionary\&edition=es-ii\&format $=\mathrm{html}$

Giddens A. (1998). Sociedad de riesgo: el contexto de la política británica. en Estudios demográficos y urbanos, 13(3). México, El Colegio de México.

Giddens A. (2000). El mundo desbocado. Taurus, España.

Huerta, Juan. (2012). "El rol de la educación en la movilidad social de México y Chile: ¿La desigualdad por otras vías?”. Revista Mexicana de Investigación educativa. 17(52) ene./mar. México.

Instituto Nacional de Estadística y Geografía. (INEGI). (2019). Consultado el 22 de febrero de 2019 en https://www.inegi.org.mx/programas/ ccpv/2020/default.html

Instituto Nacional para la Evaluación de la Educación. (INEE). (2019). Consultado el 22 de febrero de 2019 en http://publicaciones.inee.edu. $\mathrm{mx} /$ buscadorPub/P1/B/116/P1B116.pdf

Laval, Christian y Pierre, Dardot. (2013). La nueva razón del mundo. Barcelona, Gedisa Editorial.

Lu, Y. y Qin, L. (2014). "Healthy migrant and salmon bias hypothesis: A study of health and internal migration in China". Social Science \& Medicine, 102, 41-48.

Marina, José Antonio. (2010). Las culturas fracasadas. El talento y la estupidez de las sociedades. Barcelona, Anagrama.

Partida, V. y Martínez, M. (2006). “Migración Interna”. En Consejo Nacional de Población, La Situación Demográfica de México 2006 .México.

Poot, Jaques, Brigitte Waldorf y Leo Van Wissen. (2008). Migration and Human Capital.

Rodríguez, J.y Busso G. (2009). "Migración interna y desarrollo en América Latina entre 1980 y 2005". Un estudio comparativo con perspectiva regional basado en siete países. Santiago de Chile: Comisión Económica para América Latina y el Caribe ONU.

Sobrino, J. (2010). Migración interna en México durante el siglo XX. México: CONAPO.

UNAM. (s/f). Estadísticas históricas de empleo. Consultado el 25 de enero de 2019 en www.economia.unam.mx/pensiones/basesdedatos/Empleo.xls

Vargas, Mario. (2018). El llamado de la tribu, México. Alfaguara. 
Yaschine, Iliana. (2015). “¿Alcanza la educación para salir de la pobreza? Análisis del proceso de estratificación ocupacional de jóvenes rurales en México". Revista Mexicana de Ciencias Políticas y Sociales, 60(223). México.

\section{RESUMEN CURRICULAR DE LOS AUTORES}

José Antonio Soberón Mora

Maestro en Demografía por el Colegio de la Frontera Norte, Doctor en Urbanismo por la Universidad Autónoma del Estado de México. Sus líneas de investigación: Migración interna e Internacional, Dinámica Demográfica.

Dirección electrónica: josesoberon@hotmail.com

Alfonso Mejía Modesto

Maestro en Demografía por el Colegio de México, Doctor en Ciencias Políticas y Sociales por la UNAM. Ha sido profesor de asignatura en la ENAH, el ITAM y la UNAM. Profesor de tiempo completo de la UAEM desde el año 2000. Miembro del SNI de CONACYT Nivel I. Temas de investigación: Dinámica Demográfica, Urbanismo y Género.

Dirección electrónica: mejiaalfonso@yahoo.com.mx

Hugo Montes de Oca Vargas

Maestro en Demografía por el Colegio de la Frontera Norte, Estudios de Doctorado en Estudios de la Población por el Colegio de México. Perfil PRODEP. Líneas de investigación: Envejecimiento Demográfico, Envejecimiento y hogares, Envejecimiento y seguridad social y Envejecimiento y migración.

Dirección electrónica: hmontesdeocav@uaemex.mx 